\title{
Decreased Performance and Return to Play Following Anterior Cruciate Ligament Reconstruction in National Football League Wide Receivers
}

\author{
Colin J. Burgess, D.O., Erik Stapleton, D.O., M.S., Kenneth Choy, B.A., \\ Cesar Iturriaga, M.D., and Randy M. Cohn, M.D.
}

\begin{abstract}
Purpose: To identify the time to return to play (RTP) and evaluate the performance level in wide receivers in the National Football League following anterior cruciate ligament (ACL) reconstruction. Methods: A total of 29 wide receivers in the National Football League who underwent ACL reconstruction between 2013 and 2017 who met inclusion criteria were retrospectively identified and reviewed. For each player, a matched control with similar demographics was identified to compare various in-game performance measurements and seasons played. Results: Of the wide receivers that met the inclusion criteria, 9 of $29(31 \%)$ did not RTP in a regular season game following ACL reconstruction. For players who did RTP, 20 of $29(69 \%)$, the average time was 10.9 months (331.4 \pm 41.6 days). When we compared the tear group with the matched control cohort, players with ACL tears ended their careers on an average of 1.9 seasons earlier (2.2 vs 4.1 seasons, $P<.001)$ and also played less than half the number of games ( 25.5 vs 56.6 games, $P=.001$ ), respectively. Those that RTP also saw decreased performance statistics in targets (353.6 vs $125.2 P<.001)$, receptions $(208.0$ vs $74.4, P=.001)$, receiving yards (2691.0 vs 987.9, $P=.001)$, and touchdowns (17.4 vs $6.2, P=.002)$. Conclusions: Sixty-nine percent of wide receivers who underwent ACL reconstruction were able to RTP at an average of 10.9 months, or 331.4 days. Despite the majority of players being able to RTP, there was a significant decrease in both statistical performance and career duration. Level of Evidence: Level III, case-control study.
\end{abstract}

I $\mathrm{t}$ is estimated that there are more than 200,000 Anterior cruciate ligament (ACL) injuries in the United States per year. ${ }^{1}$ Clinical outcome following ACL reconstruction is most significant in professional athletes, where performance can dictate financial compensation and career longevity.

From the Donald and Barbara Zucker School of Medicine at Hofstral Northwell, Plainview (C.J.B., E.S., C.I., R.M.C.); and College of Osteopathic Medicine, New York Institute of Technology, Old Westbury (K.C.), New York, U.S.A.

The authors report that they have no conflicts of interest in the authorship and publication of this article. Full ICMJE author disclosure forms are available for this article online, as supplementary material.

The abstract of this article was presented as a virtual presentation at Southern Orthopaedic Association in June 2020.

Received June 12, 2020; accepted October 28, 2020.

Address correspondence to Colin J. Burgess, D.O., 888 Old Country Rd., Plainview, NY11803.E-mail: CBurgess1@Northwell.edu

(C) 2020 THE AUTHORS. Published by Elsevier Inc. on behalf of the Arthroscopy Association of North America. This is an open access article under the CC BY-NC-ND license (http://creativecommons.org/licenses/by-nc-nd/4.0/). 2666-061X/201030

https://doi.org/10.1016/j.asmr.2020.10.012
ACL tears account for $2 \%$ of all injuries in the $\mathrm{Na}$ tional Football League (NFL). ${ }^{2}$ In NFL players who sustain an ACL tear, it has been shown that between $8 \%$ to $37 \%$ do not return to play (RTP) in a regular season game. ${ }^{3,4}$ These RTP rates are partially dependent on position played, with skill position players having a greater RTP than unskilled players. ${ }^{5}$ Dodson et al. ${ }^{6}$ demonstrated that receivers and backs (halfbacks, fullbacks, and linebackers) were at a significantly greater injury risk than other position players.

When compared with other procedures, ACL reconstruction, along with patellar and Achilles tendon repairs, have the greatest effects on an NFL athlete's career. $^{7}$ These athletes had statistically significant decreases in games played one year after their surgery. In addition, a similar study looking at outcomes following ACL reconstruction in the four major sports (football, baseball, hockey, and basketball) found that NFL players had the shortest postinjury careers and greatest decrease in performance. ${ }^{8}$

The purpose of this study was to identify the time to RTP and evaluate the performance level in NFL wide receivers following ACL reconstruction. We 
hypothesized that because of the specific positional athletic demands, wide receivers who undergo ACL reconstruction would show a significant decrease in statistical performance and career length.

\section{Methods}

NFL wide receivers were identified using public injury data, from team releases, NFL injury reports, press releases, and other internet resources, as demonstrated by previous studies., $3,8-11$ The term "ACL injury" was used as a basis for our search. This data is publicly available; therefore, no formal institutional review board approval was required. Wide receivers who sustained an isolated, unilateral ACL injury between the 2013 and 2017 NFL seasons were evaluated. Many public injury reports specified if the ACL injury was isolated versus if additional injuries were sustained; however, it cannot be stated with complete certainty that all ACL injuries were truly isolated. Players must have participated in at least one regular season NFL game before injury to be included. Wide receivers were excluded if they had a previous ACL reconstruction, reported concomitant ligamentous/meniscal injuries in the ipsilateral knee, or other reported simultaneous injuries. The subset of players who were able to RTP were further analyzed to identify statistical performance following RTP. RTP was defined as participation in one NFL regular season game following ACL reconstruction. A player did not RTP if they only played in a preseason game, played in another football league outside of the NFL, or did not play in any NFL games following reconstruction.

Demographic data obtained for the identified players included age, body mass index (BMI), date of injury, and date of RTP. Performance statistics collected were seasons played, games played, games per season, targets, targets per season, yards per target, receptions, receptions per game, yards per reception, catch percentage, receiving yards, receiving yards per game, receiving yards per season, and touchdowns. Preseason statistics were excluded. In addition, variables such as injury laterality, player draft position, and season injured were evaluated. These variables were recorded both preinjury and postinjury for each receiver. Ingame performance statistics were analyzed as an average over the years preinjury and postinjury.

Each wide receiver in the ACL tear group was matched to a player without a documented history of ACL injury based on experience level, in-game performance, and demographics as described by previous studies. ${ }^{3,4,6,8-10}$ In decreasing order of importance for matching: previous NFL seasons, individual (nonaverage) performance statistics, and BMI. Exclusion criteria for the matched control group included players with a history of ACL injury. The control players had statistically similar performance averages preinjury and preindex year as well as similar demographics. Other injuries before the ACL injury in the matched control group were not identified. The time from the start of a player's career to the time they tore their ACL was identified in the tear group. A matched index year was applied to the control group that directly correlated to the year of injury in the tear group for each player. As an example, if a receiver tore his ACL after 5 years in the NFL, the appropriately matched control player was assigned an index year of 5 . This example control would have preindex year statistics based on years 1 through 5, and all subsequent seasons would be used for postindex year statistics.

The preinjury performance variables of players in the ACL tear group who were able to RTP were compared with the preinjury statistics of players who did not return. Then, further regression analysis was done to determine player risk factors for no RTP after ACL reconstruction. In addition, of the players who were able to RTP, preinjury performance was compared with postinjury performance measures. Lastly, the postinjury performances in the tear group were compared with the postindex variables in the matched control group. A power analysis was performed for sample size estimation based on Wise and Gallo ${ }^{12}$ comparing performance statistics for wide receivers and tight ends for postinjury to postindex controls in the National Collegiate Athletic Association Football Bowl Subdivision. With an alpha of 0.05 and power of 0.80 , the projected sample size needed for a between group comparison would be $\mathrm{n}=$ 12 (6 per group) based on difference in receptions per game and $n=16$ ( 8 per group) based on difference in receiving yards per game. Our sample size for performance comparison between groups of $n=40$ (20 per group) would be adequate for the primary aim of our study and should allow for appropriate comparison of additional performance statistics.

All statistical analyses were performed with SPSS, version 26 (IBM Corp., Armonk, NY). Patient demographics and in-game statistics of ACL tear players, who were and were not able to RTP, were compared using independent samples $t$ test for continuous variables, and the Pearson $\chi^{2}$ analyses or Fisher exact test for categorical variables. Further logistic regression was performed to determine independent risk factors of no RTP. Then, demographics and in-game statistics for players who returned to play after ACL reconstruction were compared with controls using paired samples $t$ test. Tests were deemed significant with a $P$ value less than .05. Holm-Bonferroni correction determined an adjusted significant $P$ value for regression analysis.

\section{Results}

We identified a total of 43 wide receivers who suffered a torn ACL between the 2013 and 2017 seasons. Six players had not played a regular season NFL game 
Table 1. Demographic and Preinjury Performance Data for Wide Receivers Who Returned to Play Compared With Those With No Return to Play

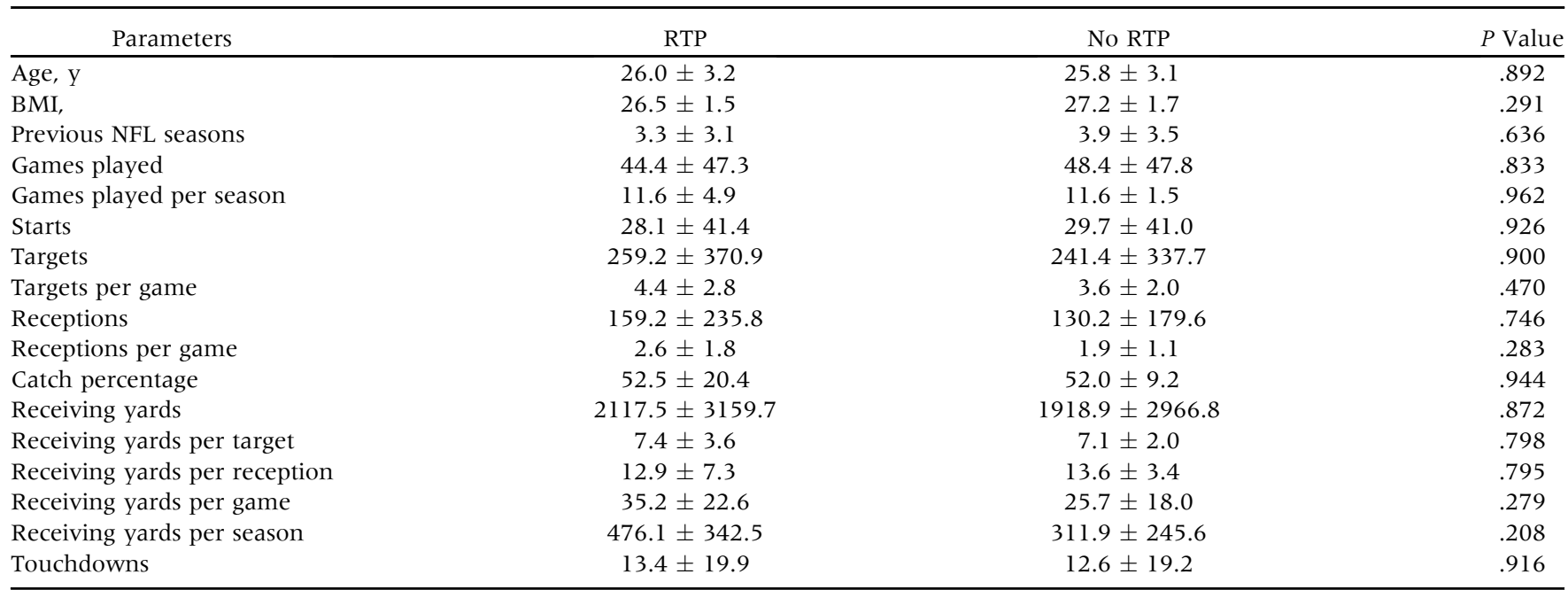

NOTE. Values are mean \pm standard deviation.

BMI, body mass index; NFL, National Football League. RTP, return to play.

before their injury. Three players had previously torn the same or contralateral ACL in their NFL career. An additional 5 players were excluded due to concomitant injuries suffered at the time of ACL tear (i.e., ACL/ posterior cruciate ligament with or without MCL/lateral collateral ligament injury as well). After we excluded these 14 players, the remaining 29 were included in this study. Of the included 29 players, $20(69.0 \%)$ were able to RTP in an NFL game after their injury. Nine $(31 \%)$ did not RTP following their ACL reconstruction. The receivers who were able to return did so at a mean of 10.9 months ( $331.4 \pm 41.6$ days) following their injury. Eleven of the $29(37.9 \%)$ tore their ACL in the preseason. The ACL group that returned to play had similar ages ( 26.0 vs 25.8 years), BMI ( 26.5 vs 27.2 ), and previous seasons of experience (3.3 vs 3.9) (Table 1). Both groups also had similar injury and draft data, with $75 \%$ of the wide receivers who RTP were drafted compared with $77.8 \%$ of those unable to RTP. There were no significant differences in any demographic, injury, or preinjury performance statistics between the ACL tear group that were able to RTP compared with the ACL tear group who did not return (Table 2). A multivariate logistic regression was performed to identify independent risk factors for no RTP. Age (odds ratio 0.11 ; 95\% confidence interval 0.02$0.72 ; P=.022$ ) and previous NFL seasons (odds ratio $10.51 ; 95 \%$ confidence interval 1.43-77.04, $P=.021$ ) approached but did not reach statistical significance as risk factors for no RTP (Table 3).

When compared with the preindex year control cohort before ACL reconstruction, wide receivers demonstrated no significant differences among parameters matched for experience, individual in-game performance, and BMI; however, there were differences in age and receiving yards per season between the 2 groups (Table 4). The preindex and postindex ingame statistics were compared for the control players, which demonstrated variable differences in almost all individual and average in-game performances, but these did not reach statistical significance (Table 5).

There were pervasive decreases in the in-game performance statistics of the ACL reconstruction cohort following RTP, with a considerable drop in receiving yards (2117.5 vs 987.9 yards, $P=.121)$. Nevertheless, no significant differences were present between preinjury and postinjury in-game in all in-game statistics for the wide receiver who underwent ACL reconstruction (Table 6).

Table 2. Injury and Draft Data for Wide Receivers Who Returned to Play Compared With Those With No Return to Play

\begin{tabular}{lrlr}
\hline \multicolumn{1}{c}{ Parameters } & RTP & No RTP & $P$ Value \\
\hline Right laterality & $9(45.0)$ & $3(33.3)$ & .694 \\
Drafted & $15(75.0)$ & $7(77.8)$ & 1.000 \\
Draft round & & & .643 \\
$\quad$ lst & $3(15.0)$ & $0(0.0)$ & \\
2nd & $5(25.0)$ & $3(33.3)$ & \\
3rd and above & $7(35.0)$ & $4(44.4)$ & .683 \\
Undrafted & $5(25.0)$ & $2(22.2)$ & \\
Season injured & $7(35.0)$ & $5(55.6)$ & \\
2013 & $3(15)$ & $0(0.0$ & \\
2014 & $4(20.0)$ & $2(22.2)$ & \\
2015 & $2(10.0)$ & $1(11.1)$ & \\
2016 & $4(20.0)$ & $1(11.1)$ & \\
2017 & & & \\
\hline NOTE. Values are $\mathrm{n}(\%)$ & & & \\
RTP, return to play. & & &
\end{tabular}


Table 3. Multivariate Logistic Regression Evaluating Demographic and Injury Parameters as Risk Factors for No Return to Play

\begin{tabular}{|c|c|c|c|}
\hline \multirow[b]{2}{*}{ Parameters } & \multicolumn{2}{|c|}{ Independent Risk of No Return to Play } & \multirow[b]{2}{*}{$P$ Value } \\
\hline & Odds Ratio & $95 \% \mathrm{CI}$ & \\
\hline Age, $y^{*}$ & 0.11 & $0.02-0.72$ & .022 \\
\hline $\mathrm{BMI}^{*}$ & 2.32 & $0.98-5.51$ & .055 \\
\hline Right laterality & 3.25 & $0.32-33.00$ & .319 \\
\hline Draft round* & 2.44 & $1.06-5.62$ & .037 \\
\hline $\begin{array}{c}\text { Previous NFL } \\
\text { seasons* }\end{array}$ & 10.51 & $1.43-77.04$ & .021 \\
\hline
\end{tabular}

BMI, body mass index; CI, confidence interval; NFL, National Football League.

*Treated as continuous variable in regression. Holm-Bonferroni correction determines new significant $P<.01$. Regression model demonstrated significance with a $\chi^{2}$ of $12.12(P=.026)$, explaining $50.0 \%$ of the variance and correctly classifying $86.2 \%$ of cases.

When comparing the post-ACLR group with matched post-index year controls, athletes showed significant decreases in subsequent participation and in-game effectiveness. Following ACL reconstruction, wide receivers played 1.9 seasons less ( 2.2 vs 4.1 seasons, $P<$ $.001)$ and less than half the number of games (25.5 vs 56.6 games, $P<.001)$ when compared with their postindex year matched controls. In addition, there were significant decreases in targets, receptions, receiving yards, and touchdowns after undergoing ACL reconstruction (Table 7).

\section{Discussion}

From our results, of the NFL wide receivers who underwent ACL reconstruction and were able to RTP, they had evidence for a significant reduction in performance and career length as compared with matched controls. These results were most notable in seasons and games played, as well as receiving yards and touchdowns. Interestingly, while player's performance averages dropped post-ACL reconstruction, these decreases were not statistically significant when comparing pre- and post-ACL reconstruction in players who RTP. This can possibly be explained by the partial bias of patients selected for analysis. It has been shown that players with 4 years or more of experience have greater RTP percentage after ACL reconstruction. ${ }^{4}$ In our cohort of players who RTP, there were $30 \%$ that had 4 years or more experience. However, there was no statistically significant difference in games and seasons played between the RTP and non-RTP groups. Results after ACL reconstruction can be variable, with poor results having the most detrimental effect on professional athletes mentally, physically, and financially. Despite the constant advancement in reconstruction techniques, based on this study, $31 \%$ of NFL wide receivers are unable to RTP in an NFL game after surgery.

Our findings indicate that when compared with matched controls, wide receivers who undergo ACL reconstruction do have a significant decrease in certain performance measures and career duration. Wide receivers post-ACL reconstruction played fewer games, games per season, and total seasons compared with matched controls. This data appears plausible, as wide receivers frequently perform explosive pivoting maneuvers in their route running, and this increased repetitive stress on the knee could predispose them to more risk for ACL injury. This data coincides with previous studies on athletes in the NFL as well as other professional sports. One study demonstrated that up to $40 \%$ of NFL players were no longer on an active team roster after just 3 seasons post-ACL reconstruction. ${ }^{8}$ This same study compared professional athletes in the NFL, National Basketball Association, National Hockey

Table 4. Wide Receiver Baseline Comparison: Pre-ACLR Cohort Versus Preindex Control

\begin{tabular}{|c|c|c|c|}
\hline Age, y & $26.0 \pm 3.2$ & $25.3 \pm 3.2$ & .023 \\
\hline BMI & $26.5 \pm 1.5$ & $26.5 \pm 1.3$ & .918 \\
\hline Previous NFL seasons & $3.3 \pm 3.1$ & $3.3 \pm 3.1$ & 1.000 \\
\hline Games played per season & $11.6 \pm 4.9$ & $12.0 \pm 4.4$ & .525 \\
\hline Targets & $259.2 \pm 370.9$ & $304.6 \pm 364.8$ & .175 \\
\hline Targets per game & $4.4 \pm 2.8$ & $5.5 \pm 2.9$ & .056 \\
\hline Catch percentage & $52.5 \pm 20.4$ & $52.2 \pm 19.2$ & .872 \\
\hline Receiving yards & $2117.5 \pm 3159.7$ & $2490.8 \pm 3124.5$ & .107 \\
\hline Receiving yards per target & $7.4 \pm 3.6$ & $6.9 \pm 2.7$ & .533 \\
\hline Receiving yards per reception & $12.9 \pm 7.3$ & $12.1 \pm 4.9$ & .604 \\
\hline Receiving yards per game & $35.2 \pm 22.6$ & $43.6 \pm 25.1$ & .049 \\
\hline Receiving yards per season & $476.1 \pm 342.5$ & $610.1 \pm 364.7$ & .040 \\
\hline
\end{tabular}

NOTE. Values are mean \pm standard deviation.

ACLR, anterior cruciate ligament reconstruction; BMI, body mass index; NFL, National Football League. 
Table 5. Wide Receiver Index Comparison: Preindex Cohort Versus Postindex Cohort

\begin{tabular}{lccc}
\hline \multicolumn{1}{c}{ Parameters } & Preindex & Postindex & $P$ Value \\
\hline NFL seasons & $3.3 \pm 3.1$ & $4.1 \pm 2.3$ & .420 \\
Games played & $45.2 \pm 42.6$ & $56.6 \pm 34.1$ & .392 \\
Games played per season & $12.0 \pm 4.4$ & $13.3 \pm 2.4$ & .295 \\
Targets & $304.6 \pm 364.8$ & $353.6 \pm 305.6$ & .611 \\
Targets per game & $5.5 \pm 2.9$ & $5.7 \pm 2.3$ & .709 \\
Receptions & $182.4 \pm 217.4$ & $208.0 \pm 184.8$ & .657 \\
Receptions per game & $3.2 \pm 1.7$ & $3.4 \pm 1.4$ & .668 \\
Catch percentage & $52.2 \pm 19.2$ & $59.5 \pm 8.1$ & .089 \\
Receiving yards & $2490.8 \pm 3124.5$ & $2691.0 \pm 2275.4$ & .803 \\
Receiving yards per target & $6.9 \pm 2.7$ & $7.8 \pm 0.9$ & .153 \\
Receiving yards per reception & $12.1 \pm 4.9$ & $13.3 \pm 2.0$ & .248 \\
Receiving yards per game & $43.6 \pm 25.1$ & $44.7 \pm 19.3$ & .819 \\
Receiving yards per season & $610.1 \pm 364.7$ & $598.2 \pm 286.4$ \\
Touchdowns & $16.8 \pm 21.1$ & $17.4 \pm 15.5$ \\
\hline
\end{tabular}

NOTE. Values are mean \pm standard deviation.

NFL, National Football League.

League, and Major League Baseball. All athletes in these 4 sports who underwent ACL reconstruction played a statistically significant fewer number of games after the surgery. ${ }^{8}$ In contrast, one study exhibited no statistically significant difference in career length following ACL reconstruction in NFL quarterbacks. ${ }^{3}$ A similar study also showed no difference in career length following ACL reconstruction in NFL players; however, when combined with meniscectomy there was a statistically significant decrease in games and years played. ${ }^{13}$

Carey et al. ${ }^{14}$ performed a similar analysis of RTP and performance following ACL reconstruction in NFL wide receivers and running backs. They had similar results to our findings, demonstrating a $79 \%$ RTP rate. They also found an approximately $33 \%$ decrease in performance following ACL reconstruction, which was statistically significant. While our results were not statistically significant when comparing pre- and postinjury statistics, they did demonstrate a similar decrease in performance. This lack of statistical significance in our study could be a result of analyzing individual statistics as opposed to a consolidated measure of performance. In addition, their control group consisted of more than 4 times more players than their injury group, potentially allowing for a more generalizable comparison of postinjury statistics to the average player postindex year. Our study focused on individually matched controls for each player undergoing ACL reconstruction and thus potentially limited the generalizability throughout NFL wide receivers. Nevertheless, comparing unvalidated markers of performance instead of performance statistics may have introduced bias and their power rating comparison only reflects a difference in yards and touchdowns.

A multivariate analysis was performed on player demographics who were unable to RTP following ACL reconstruction. To account for cumulative type I error in our tests of multiple hypotheses, Holm-Bonferroni method was used to set new threshold $P$ value for statistical significance at .01 . None of the variables tested were statistically significant; however, number of

Table 6. Wide Receiver ACLR Comparison: Pre-ACLR Cohort Versus Post-ACLR Cohort

\begin{tabular}{lccc}
\hline \multicolumn{1}{c}{ Parameters } & Pre-ACLR & Post-ACLR & $P$ Value \\
\hline NFL seasons & $3.3 \pm 3.1$ & $2.2 \pm 1.2$ & .191 \\
Games played & $44.4 \pm 47.3$ & $25.5 \pm 19.4$ & .126 \\
Games played per season & $11.6 \pm 4.9$ & $10.7 \pm 4.5$ & .495 \\
Targets & $259.2 \pm 370.9$ & $125.2 \pm 129.2$ & .118 \\
Targets per game & $4.4 \pm 2.8$ & $4.3 \pm 2.8$ & .893 \\
Receptions & $159.2 \pm 235.8$ & $74.4 \pm 79.1$ & .114 \\
Receptions per game & $2.6 \pm 1.8$ & $2.6 \pm 1.9$ & .903 \\
Catch percentage & $52.5 \pm 20.4$ & $58.1 \pm 13.3$ & .198 \\
Receiving yards & $2117.5 \pm 3159.7$ & $987.9 \pm 1051.9$ & .121 \\
Receiving yards per target & $7.4 \pm 3.6$ & $7.8 \pm 1.4$ & .570 \\
Receiving yards per reception & $12.9 \pm 7.3$ & $14.1 \pm 3.7$ & .415 \\
Receiving yards per game & $35.2 \pm 22.6$ & $33.6 \pm 22.6$ & .660 \\
Receiving yards per season & $476.1 \pm 342.5$ & $417.5 \pm 370.7$ & $.3 .2 \pm 7.5$ \\
Touchdowns & $13.4 \pm 19.9$ & $6.2 \pm 3$
\end{tabular}

NOTE. Values are mean \pm standard deviation.

ACLR, anterior cruciate ligament reconstruction; NFL, National Football League. 
Table 7. Wide Receiver Post-Comparison: Post-ACLR Cohort Versus Postindex Cohort

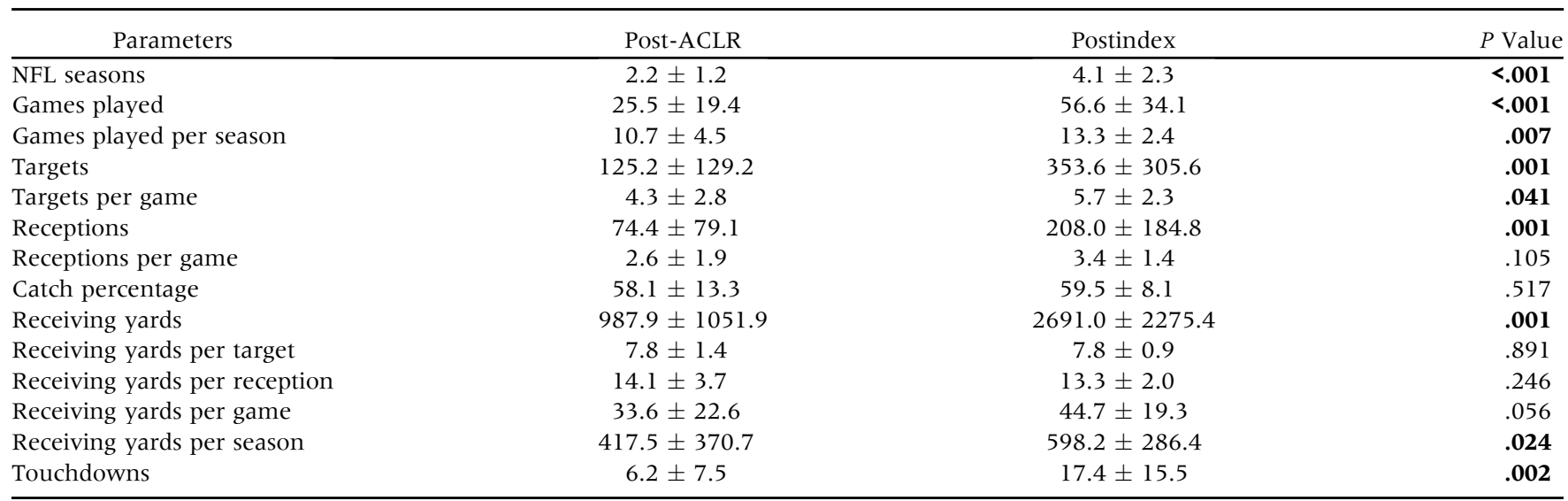

NOTE. Values are mean \pm standard deviation.

Bold values indicate statistical significance.

ACLR, anterior cruciate ligament reconstruction; NFL, National Football League.

seasons played before injury approached statistical significance. For each year played before injury, players were at 10.51 times increased likelihood of not returning to play. This contradicts other studies demonstrating an increased percentage in RTP for players with 4 or more years of experience before injury. ${ }^{4}$ In future studies with an increased database of players, further data can be explored to determine whether this is truly a risk factor for decreased RTP.

Reduction in performance for NFL players after ACL reconstruction has been reported for defensive players, ${ }^{11}$ and this study found decreases in games started as well as solo tackles in players who underwent ACL reconstruction. In contrast, Cinque et al. ${ }^{9}$ showed that in NFL linemen who underwent ACL reconstruction, there were no significant differences in performance after surgery. A similar study by Erickson et al. ${ }^{3}$ looked at performance in NFL quarterbacks after ACL reconstruction did not find statistically significant differences in pre-injury versus post injury performance as compared to controls. They did, however, show similar results to our study such that there were no significant differences in statistics before and after ACL reconstruction among players who RTP.

Results from this study can have a measurable impact on the dynamics of the NFL, from statistical and performance predictions to various personnel and managerial decisions. Potential prediction of player performance after surgery can have huge financial implications as well as help team administration provide a good baseline for expected athlete's performance after surgery. A study by Secrist et al. ${ }^{15}$ demonstrated a decrease in earnings following ACL reconstruction in NFL players during the 4 years after surgery. They also demonstrated that players earning more than $\$ 2$ million per year before injury did not have negatively impacted careers versus those earning less than $\$ 2$ million. The livelihoods of these players without larger contracts can be negatively affected by ACL injuries. This difference could partially be attributed to the higher-earning players having a more impactful role as opposed to the lower-earning players.

Future studies can further evaluate differences based on reconstruction technique as well as additional comparison across various positions. In addition, financial implications after injury can be further explored, which can help give teams a better understanding of player outcomes after surgical reconstruction. Developing a predictive model for performance after ACL reconstruction throughout NFL players would be beneficial for teams, personnel and players themselves.

\section{Limitations}

There are a few limitations noted by the authors. Due to the retrospective nature of the study, there are inherent flaws with data acquisition, although the authors used similar methodology described from previous studies., ${ }^{3,4,8-11}$ Second, while the injuries were made public, full disclosure of the exact severity and nature of the injuries as well as surgical technique is not known. NFL teams do not disclose details regarding player injuries to the public. In addition, the authors were not able to attain the operative notes or surgical methods used for each ACL reconstruction. Therefore, accounting for different surgical details (i.e., autograft vs allograft, graft choice, surgical techniques) was not able to be accomplished in this study. In addition, we also did not look at whether these injuries occurred during practice or games; however, it has been shown that the majority of injuries in the NFL occur during games and we recorded this as such. ${ }^{16}$ When 
performing our multivariate analysis to determine factors associated with decreased RTP, our study population consisted of only 9 players, limiting the power of our results. Nevertheless, our study was adequately powered to determine differences between post-ACL reconstruction and post-index groups performance based on previous literature demonstrating distinct receptions and yards per game among similar groups in National Collegiate Athletic Association receivers. ${ }^{12}$

\section{Conclusions}

Sixty-nine percent of wide receivers who underwent ACL reconstruction were able to RTP at an average of 10.9 months, or 331.4 days. Despite the majority of players being able to RTP, there was a significant decrease in both statistical performance and career duration.

\section{References}

1. Gordon MD, Steiner M. Anterior cruciate ligament injuries. In: Garrick JG, ed. Orthopaedic knowledge update sports medicine 3. Rosemont, IL: American Academy of Orthopaedic Surgeons, 2004.

2. Bradley JP, Klimkiewicz JJ, Rytel MJ, Powell JW. Anterior cruciate ligament injuries in the National Football League: Epidemiology and current treatment trends among team physicians. Arthroscopy 2002;18:502-509.

3. Erickson BJ, Harris JD, Heninger JR, et al. Performance and return-to-sport after ACL reconstruction in NFL quarterbacks. Orthopedics 2014;37.

4. Shah VM, Andrews JR, Fleisig GS, McMichael CS, Lemak LJ. Return to play after anterior cruciate ligament reconstruction in National Football League athletes. Am J Sports Med $2010 ; 38: 2233-2239$.

5. Yang J, Hodax JD, Machan JT, et al. National Football League skilled and unskilled positions vary in opportunity and yield in return to play after an anterior cruciate ligament injury. Orthop J Sports Med 2017;5.

6. Dodson CC, Secrist ES, Bhat SB, Woods DP, Deluca PF. Anterior cruciate ligament injuries in National Football League athletes from 2010 to 2013: A descriptive epidemiology study. Orthop J Sports Med 2016;4.
7. Mai HT, Alvarez AP, Freshman RD, et al. The NFL orthopaedic surgery outcomes database (NO-SOD): The effect of common orthopaedic procedures on football careers. Am J Sports Med 2016;44:2255-2262.

8. Mai HT, Chun DS, Schneider AD, et al. Performancebased outcomes after anterior cruciate ligament reconstruction in professional athletes differ between sports. Am J Sports Med 2017;45:2226-2232.

9. Cinque ME, Hannon CP, Bohl DD, et al. Return to sport and performance after anterior cruciate ligament reconstruction in National Football League linemen. Orthop $J$ Sports Med 2017;5.

10. Eisenstein ED, Rawicki NL, Rensing NJ, Kusnezov NA, Lanzi JT. Variables affecting return to play after anterior cruciate ligament injury in the National Football League [published online October 25, 2016]. Orthop J Sports Med. https://doi.org/10.1177/2325967116670117.

11. Read CR, Aune KT, Cain EL, Fleisig GS. Return to play and decreased performance after anterior cruciate ligament reconstruction in National Football League defensive players. Am J Sports Med 2017;45:1815-1821.

12. Wise PM, Gallo RA. Impact of anterior cruciate ligament reconstruction on NCAA FBS football players: Return to play and performance vary by position. Orthop J Sports Med 2019;7:1-6.

13. Brophy RH, Gill CS, Lyman S, Barnes RP, Rodeo SA, Warren RF. Effect of anterior cruciate ligament reconstruction and meniscectomy on length of career in National Football League athletes: A Case control Study. Am J Sports Med 2009;37:2102-2107.

14. Carey JL, Huffman GR, Parekh SG, Sennett BJ. Outcomes of anterior cruciate ligament injuries to running backs and wide receivers in the National Football League. Am J Sports Med 2006;34:1911-1917.

15. Secrist ES, Bhat SB, Dodson CC. The financial and professional impact of anterior cruciate ligament injuries in National Football League athletes [published online August 30, 2016]. Orthop J Sports Med. https://doi.org/10. $1177 / 2325967116663921$.

16. Lawrence DW, Hutchison MG, Comper P. Descriptive epidemiology of musculoskeletal injuries and concussions in the National Football League, 2012-2014. Orthop J Sports Med 2015;3:2012-2014. 